\title{
Nanogenerators for Self-Powered Flexible Electronics and Piezotronics for Active Human-Machine Interfacing
}

\author{
Zhong Lin Wang \\ School of Materials Science and Engineering, Georgia Institute of Technology \\ Atlanta USA \\ Beijing Institute of Nanoenergy and Nanosystems, Chinese Academy of Sciences \\ Beijing, China
}

Developing wireless nanodevices and nanosystems is of critical importance for sensing, medical science, environmental/infrastructure monitoring, defense technology and even personal electronics. It is highly desirable for wireless devices to be self-powered without using battery. Nanogenerators (NGs) have been developed based on piezoelectric, trioboelectric and pyroelectric effects, aiming at building self-sufficient power sources for mico/nano-systems. The output of the nanogenerators now is high enough to drive a wireless sensor system and charge a battery for a cell phone, and they are becoming a vital technology for sustainable, independent and maintenance free operation of micro/nano-systems and mobile/portable electronics. An energy conversion efficiency of 55\% and an output power density of $500 \mathrm{~W} / \mathrm{m}^{2}$ have been demonstrated. This technology is now not only capable of driving portable electronics, but also has the potential for harvesting wind and ocean wave energy for large-scale power application. This talk will focus on the updated progress in NGs.

For Wurtzite and zinc blend structures that have non-central symmetry, such as $\mathrm{ZnO}, \mathrm{GaN}$ and $\mathrm{InN}$, a piezoelectric potential (piezopotential) is created in the crystal by applying a strain. Such piezopotential can serve as a "gate" voltage that can effectively tune/control the charge transport across an interface/junction; electronics fabricated based on such a mechanism is coined as piezotronics, with applications in force/pressure triggered/controlled electronic devices, sensors, logic units and memory. By using the piezotronic effect, we show that the optoelectronc devices fabricated using wurtzite materials can have superior performance as solar cell, photon detector and light emitting diode. Piezotronics is likely to serve as a "mechanosensation" for directly interfacing biomechanical action with silicon based technology and active flexible electronics. This lecture will focus on the updated progress in the field and its expansion to 2D materials.

\section{References}

[1] Z. L. Wang "Triboelectric Nanogenerators as New Energy Technology for Self-Powered Systems and as Active Mechanical and Chemical Sensors," ACS Nano, vol. 7, pp. 9533-9557, 2013.

[2] G. Zhu, J. Chen, T. Zhang, Q. Jing, and Z. L. Wang, "Radial-arrayed rotary electrification for high-performance triboelectric generator," Nature Communication, vol. 5, pp. 3456, 2014.

[3] Z. L. Wang, J. Chen, and L. Lin, "Progress in triboelectric nanogenertors as new energy technology and self-powered sensors," Energy \& Environmental Sci, vol. 8, pp. 2250-2282, 2015.

[4] W. Z. Wu, X. N. Wen, and Z. L. Wang, "Pixel-addressable matrix of vertical-nanowire piezotronic transistors for active/adaptive tactile imaging," Science, vol. 340, pp. 952-957, 2013.

[5] C. F. Pan, L. Dong, G. Zhu, S. Niu, R. Yu, Q. Yang, Y. Liu, and Z. L. Wang, "Micrometer-resolution electroluminescence parallel-imaging of pressure distribution using piezoelectric nanowire-LED array," Nature Photonics, vol. 7, pp. 752-758, 2013.

[6] W. Z. Wu, L. Wang, Y. L. Li, F. Zhang, L. Lin, S. Niu, D. Chenet, X. Zhang, Y. Hao, T. F. Heinz, J. Hone, and Z. L. Wang, "Piezoelectricity of single-atomic-layer $\mathrm{MoS}_{2}$ for energy conversion and piezotronics," Nature, vol. 514, pp. 470-474, 2014.

[7] W. Z. Wu and Z. L. Wang, "Piezotronics and piezo-phototronics for smart adaptive electronics and optoelectronics," Nature Review Materials, vol. 1, pp. 16031, 2016. 